\title{
Factors associated with treatment interruption in elderly patients with cancer
}

\author{
Hye Sung Won ${ }^{1}$, Der Sheng Sun ${ }^{1}$, Ji Young $\mathrm{Choi}^{2}$, Ho Jung An ${ }^{3}$ and Yoon Ho $\mathrm{Ko}^{1}$
}

\begin{abstract}
${ }^{1}$ Division of Medical Oncology, Department of Internal Medicine, ${ }^{2}$ Department of Nursing, College of Medicine, Uijeongbu St. Mary's Hospital, The Catholic University of Korea, Uijeongbu; ${ }^{3}$ Division of Medical Oncology, Department of Internal Medicine, College of Medicine, St. Vincent's Hospital, The Catholic University of Korea, Suwon, Korea
\end{abstract}

Received: October 4, 2016 Revised : February 20, 2017 Accepted: May 14, 2017

\section{Correspondence to}

Yoon Ho Ko, M.D.

Division of Medical Oncology, Department of Internal Medicine, College of Medicine, Uijeongbu St. Mary's Hospital, The Catholic University of Korea, 271 Cheonbo-ro, Uijeongbu 11765 , Korea

Tel: $+82-31-820-3985$

Fax: $+82-31-847-2719$

E-mail: koyoonho@catholic.ac.kr
Background/Aims: This study was conducted to identify risk factors that predict vulnerability to cancer therapy on the basis of the clinical, geriatric, and quality of life assessment before starting treatment in elderly patients.

Methods: Seventy-five patients aged 65 years and over with newly diagnosed stage IV solid cancer receiving chemotherapy were analyzed. Clinical and laboratory data were collected. The geriatric assessment was performed using the Korean versions of the Modified Barthel Index, Instrumental Activities of Daily Living, Mini-Mental State Examination, and Geriatric Depression Scale. The European Organisation for Research and Treatment of Cancer Quality-of-Life Core Questionnaire (EORTC-QLQ-C30) was also performed.

Results: Forty-one patients stopped cancer treatment during or after the end of first-line therapy and were classified as the treatment interruption group. By univariate analysis, treatment interruption was associated with metastases to $\geq 2$ distant sites, lower albumin level, lower EORTC-QLQ-C30 physical and role functioning scores, and higher EORTC-QLQ-C30 fatigue and appetite loss symptom scores. By multivariate analysis, treatment interruption was significantly associated with low score for the EORTC-QLQ-C30 physical functioning scale (odds ratio [OR], 1.020; 95\% confidence interval [CI], 1.002 to $1.039 ; p=0.030$ ), and $\geq 2$ sites of distant metastases (OR, 2.965; 95\% CI, 1.012 to 8.681; $p=0.047$ ).

Conclusions: The EORTC-QLQ-C30 physical functioning score and metastases to $\geq 2$ organs, which indicate a poor physical functional status and metastatic high tumor burden, were significantly associated with interruption of first-line treatment in elderly patients with cancer.

Keywords: Chemotherapy; Geriatric assessment; Quality of life; Aged

\section{INTRODUCTION}

Aging populations are increasing rapidly worldwide, and Korea will not be an exception to this trend. According to data published by Statistics Korea in 2013, people aged 65 years and older comprised $12.2 \%$ of the entire population and this percentage is expected to reach $24.3 \%$ by 2030 [1]. The most common cause of death among the elderly is cancer. Thus, the management of elderly patients with cancer is an important medical problem. However, few studies have focused on the management of elderly patients with cancer, and there are few standard tools to assess the fitness of older patients for chemotherapy $[2,3]$. In clinical practice, elderly patients may be undertreated because of vague concerns about their tolerance to chemotherapy. On the other hand, elderly people do sometimes experience adverse events due to severe chemotherapy-related toxicity. 
Over the past decade, interest in the use of the comprehensive geriatric assessment (CGA) as a tool for managing elderly patients with cancer has increased gradually. CGA is defined as a multidimensional assessment of general health status that includes assessment of the medical, functional, and psychosocial capabilities of elderly patients $[2,4]$. Recent attempts have been made to use the CGA to distinguish patients who are likely to benefit from chemotherapy from those who may be vulnerable to chemotherapy [2]. However, optimum combination of geriatric assessment components for treatment decision in elderly patients with cancer has not been determined. Most studies have evaluated only geriatric assessment variables without analyzing their relationships with various clinical and laboratory parameters. It is difficult to use complicated and timeconsuming CGA tools in clinical practice, even though this would be best. Thus, we intended to use questionnaire tools as simple as possible to assess general health status for elderly patients and to evaluate various laboratory parameters simultaneously.

The aim of this study was to identify risk factors that predict vulnerability to treatment on the basis of the assessment before starting treatment in real practice. We compared the clinical and laboratory variables and geriatric assessment components between patients whose treatment was interrupted (treatment interruption group) and those who continued treatment (treatment continuation group) during first-line therapy in elderly patients with cancer.

\section{METHODS}

\section{Study schema and data collection}

Patients were enrolled according to the following inclusion criteria: (1) age 65 years or older, (2) newly diagnosed, pathologically proven solid cancer, and (3) life expectancy of $\geq 3$ months. Patients with psychiatric disorders or serious uncontrolled comorbidities or who could not communicate were excluded. Written informed consent was obtained from each patient before enrollment. We enrolled patients being treated at Uijeongbu St. Mary's Hospital between April 2012 and November 2013. The questionnaires for geriatric assessment were administered by one professional nurse before the initiation of the planned treatment. The clinical and laboratory data at the time of diagnosis before treatment were collected. The following laboratory tests were performed and the values used to evaluate the relationships with the CGA: complete blood count, serum levels of albumin, creatinine, lactate dehydrogenase (LDH), C-reactive protein (CRP), and D-dimer. The patients received treatment and were followed up until death or December 2015 . Treatment interruption group included patients who had discontinued treatment during their first-line therapy or who had not received second-line chemotherapy after progression for the following reasons: (1) physicians determined that additional treatment was not appropriate due to deterioration in general condition of the patient and (2) patients refused the continuous treatment due to treatment-related toxicity. Ethical committee approval was obtained from the Institutional Review Board of The Catholic University of Korea, Uijeongbu St. Mary's Hospital (UC12QISIoo13).

\section{Geriatric assessment}

The geriatric assessment included the following instruments: Korean version of the Modified Barthel Index (K-MBI), Korean version of Instrumental Activities of Daily Living (K-IADL), Mini-Mental State Examination (K-MMSE), Geriatric Depression Scale (K-GDS), and the European Organisation for Research and Treatment of Cancer Quality-of-Life Core Questionnaire (EORTC-QLQ-C3O). All questionnaires were previously validated in the Korean language [5-8]. First, we used the $\mathrm{K}-\mathrm{MBI}$ (SHAH version) to assess performance in activities of daily living. This comprises 10 items and yields a score of o to 100; a score of 100 is interpreted to indicate independence in activities of daily living [5]. Second, we used the K-IADL to evaluate the instrumental functions necessary for adaptation to social life. Patients with at least one dependency in IADL were classified as IADL dependent. Third, we used the K-MMSE as a screening tool for cognitive dysfunction in the elderly patients. The scores range of o to 30 . For the present study, we adopted a single cutoff and classified the range of o to 24 as definitive cognitive dysfunction $[6,9]$. Fourth, mood was assessed using the K-GDS, which comprises 30 questions and is scored range from o to 30 . Scores $\geq 16$ were classified as indicating a depressive disorder [7]. 


\section{Quality of life}

We used the EORTC-QLQ-C30 to assess the quality of life. The EORTC-QLQ-C30 (version 3.0) is a quality-oflife questionnaire used extensively in patients with cancer. It comprises 30 questions about global health status, functioning scales (physical, role, emotional, cognitive, and social), and symptom scales (fatigue, nausea/vomiting, pain, dyspnea, insomnia, appetite loss, constipation, diarrhea, and financial difficulties). Raw scores for each scale were linearly transformed into a score of o to 100. A high score for the global health status and functioning scales represents a better level of functioning and a high quality of life, whereas a high score for symptom scales represents worse symptoms [8].

\section{Statistical analysis}

Continuous variables were expressed as mean with standard deviation. Continuous variables were compared using the $t$ test or nonparametric Mann-Whitney U test. The chi-square test and Fisher exact test were used to compare categorical variables. Univariate and multivariate logistic regression analyses were performed to compare between the treatment interruption and continuation groups. To determine the significance of bivariate associations, we used Pearson correlation coefficient. Progression free survival (PFS) was defined as the time from start date of chemotherapy to disease progression or death from any cause. Overall survival (OS) was defined as start date of chemotherapy until death from any cause. Univariate survival analysis was performed using the Kaplan-Meier method, and the log-rank test was applied to identify significant differences. Multivariate survival analysis was performed using the Cox proportional-hazard regression model. Statistical analyses were performed using IBM SPSS version 19.0 (IBM Co., Armonk, NY, USA) and $p<0.05$ was considered significant.

\section{RESULTS}

\section{Patient characteristics}

We enrolled 111 elderly cancer patients. However, 20 patients with stage II or III cancer, nine patients who did not receive treatment, and seven patients who received targeted or hormone therapy were excluded from the final analysis. Therefore, 75 patients were included in this study. The general characteristics of the 75 patients are summarized in Table 1. The mean age of the patients was 72.7 years (standard deviation, 5.0). Fifty-three patients (70.6\%) had one or more comorbid disease, and the most common comorbidity was hypertension (60.0\%). The numbers of patients with diabetes, stroke, cardiovascular disease, and pulmonary disease were 15 (20.0\%), five (6.7\%), five (6.7\%), and three (4.0\%), respectively. The most frequent cancer types were gastrointestinal (28.0\%) and lung/head and neck (21.3\%) cancers. Twenty-five (33.1\%) had metastases to two or more organs, and the most common sites of distant metastases were liver (30.7\%), lung (28.0\%), distant lymph nodes (28.0\%), and bone (26.7\%).

Twenty patients (26.6\%) received monochemotherapy and 55 patients $(73.4 \%)$ received combination-chemotherapy. The median number of cycles of chemotherapy was 3 (range, 1 to 12). Forty patients (53.3\%) received fulldose chemotherapy and 19 patients $(25.3 \%)$ received a reduced starting dose of chemotherapy.

Among the 75 patients, 41 patients (54.6\%) stopped cancer treatment during the first-line therapy or after end of first-line therapy because of death, deteriorated condition, or the patient's refusal and these patients were classified as the treatment interruption group. Twenty patients stopped treatment due to death, and 10 and 11 patients discontinued therapy due to deteriorated condition and patient's refusal, respectively. The median PFS was 3.7 months (range, 0.17 to 11.4) in the treatment interruption group and 4.8 months (range, 0.77 to 34.2 ) in the continuation group.

\section{Geriatric assessment and quality of life}

The geriatric assessment and quality of life scores of patients are summarized in Table 2. The K-MBI, K-IADL, and EORTC-QLQ-C3O were administered to all patients, and the K-MMSE and K-GDS were administered to 74 patients. Thirty-seven (49.3\%) and 33 patients (44.0\%) were classified as dependent in activities of daily living and instrumental activities of daily living at the time of diagnosis, respectively. We estimated that 45 patients (60.8\%) had some degree of decline in cognitive function, and 19 patients (25.7\%) had a depressive disorder.

We evaluated the relationships between the geriatric assessment components and clinical and laboratory parameters. The K-MBI correlated negatively with 
Won HS, et al. Chemotherapy interruption in elderly patients

Table 1. Clinical and laboratory characteristics of the patients

\begin{tabular}{|c|c|c|c|c|}
\hline Parameter & All patients $(n=75)$ & Continuation $(\mathrm{n}=34)$ & Interruption $(n=41)$ & $p$ value \\
\hline Age, yr & $72.7 \pm 5.0$ & $72.3 \pm 4.9$ & $73.1 \pm 5.2$ & 0.843 \\
\hline $65-69$ & $17(22.7)$ & $10(29.4)$ & $7(17.1)$ & 0.289 \\
\hline $70-74$ & $35(46.7)$ & $12(35 \cdot 3)$ & $23(56.0)$ & \\
\hline $75-79$ & $16(21.3)$ & $9(26.5)$ & $7(17.1)$ & \\
\hline $80-84$ & $5(6.6)$ & $3(8.8)$ & $2(4.9)$ & \\
\hline $85-89$ & $2(2.7)$ & $\circ$ & $2(4.9)$ & \\
\hline Sex & & & & 0.908 \\
\hline Male & $48(64.0)$ & $22(64.7)$ & $26(63.4)$ & \\
\hline Female & $27(36.0)$ & $12(35.3)$ & $15(36.6)$ & \\
\hline Body mass index, $\mathrm{kg} / \mathrm{m}^{2}$ & & & & 0.101 \\
\hline$<23$ & $43(57 \cdot 3)$ & $16(47.1)$ & $27(65.9)$ & \\
\hline$\geq 23$ & $32(42.7)$ & $18(52.9)$ & $14(34 \cdot 1)$ & \\
\hline Weight loss in past 6 months & & & & 0.108 \\
\hline No & $55(73.3)$ & $28(82.3)$ & $27(65.9)$ & \\
\hline Yes & $20(26.7)$ & $6(17.7)$ & $14(34.1)$ & \\
\hline ECOG PS & & & & 0.811 \\
\hline o/1 & $69(92.0)$ & $31(91.2)$ & $38(92.7)$ & \\
\hline 2 & $6(8.0)$ & $3(8.8)$ & $3(7.3)$ & \\
\hline Smoking & & & & 0.094 \\
\hline Nonsmoker & $34(45 \cdot 3)$ & $12(35.2)$ & $22(53.7)$ & \\
\hline Ex- or current-smoker & $37(49.4)$ & $18(53.0)$ & $19(46.3)$ & \\
\hline Missing & $4(5 \cdot 3)$ & $4(11.8)$ & o & \\
\hline Educational level & & & & 0.803 \\
\hline Elementary school or less & $40(53.3)$ & $18(47.4)$ & $25(56.8)$ & \\
\hline Middle/high school graduate & $31(41.3)$ & $18(47.4)$ & $16(36.4)$ & \\
\hline Bachelor's degree or higher & $2(2.7)$ & $1(2.6)$ & $2(4.5)$ & \\
\hline Missing & $2(2.7)$ & $1(2.6)$ & $1(2.3)$ & \\
\hline Religion & & & & 0.559 \\
\hline No & $46(61.3)$ & $22(64.7)$ & $24(58.5)$ & \\
\hline Yes & $29(38.7)$ & $12(35.5)$ & $17(41.5)$ & \\
\hline No. of comorbidities & & & & 0.491 \\
\hline o & $22(29.3)$ & $10(29.4)$ & $12(29 \cdot 3)$ & \\
\hline 1 or 2 & $48(64.0)$ & $23(67.6)$ & $25(61.0)$ & \\
\hline 3 or more & $5(6.7)$ & $1(3.0)$ & $4(9.7)$ & \\
\hline Cancer type & & & & 0.435 \\
\hline Gastrointestinal & $21(28.0)$ & $10(29.4)$ & $11(26.7)$ & \\
\hline Lung/head and neck & $16(21.3)$ & $7(20.6)$ & $9(22.0)$ & \\
\hline Pancreaticobiliary & $17(22.7)$ & $5(14.7)$ & $12(29 \cdot 3)$ & \\
\hline Genitourinary & $11(14 \cdot 7)$ & $7(20.6)$ & $4(9.8)$ & \\
\hline Others & $10(13.3)$ & $5(14.7)$ & $5(12.2)$ & \\
\hline No. of distant metastases & & & & $0.033^{\mathrm{a}}$ \\
\hline o or 1 & $50(66.7)$ & $27(79.4)$ & $23(58.5)$ & \\
\hline
\end{tabular}


Table 1. Continued

\begin{tabular}{|c|c|c|c|c|}
\hline Parameter & All patients $(\mathrm{n}=75)$ & Continuation $(\mathrm{n}=34)$ & Interruption $(\mathrm{n}=41)$ & $p$ value \\
\hline 2 or more & $25(33.4)$ & $7(20.6)$ & $18(41.5)$ & \\
\hline Cytotoxic chemotherapy & & & & 0.111 \\
\hline Monochemotherapy & $20(26.6)$ & $6(17.6)$ & $14(34.1)$ & \\
\hline Polychemotherapy & $55(73.4)$ & $28(82.4)$ & $27(65.9)$ & \\
\hline Hemoglobin, g/dL & $11.4 \pm 1.8$ & $11.6 \pm 1.9$ & $11.2 \pm 1.7$ & 0.398 \\
\hline White blood cell, $10^{3} / \mathrm{mm}^{3}$ & $8.3 \pm 4.7$ & $8.3 \pm 5 \cdot 5$ & $8.2 \pm 3.9$ & 0.925 \\
\hline Platelet, $10^{3} / \mathrm{mm}^{3}$ & $267 \pm 88$ & $282 \pm 92$ & $255 \pm 84$ & 0.199 \\
\hline Albumin, g/dL & $3.4 \pm 0.5$ & $3.5 \pm 0.4$ & $3.2 \pm 0.6$ & $0.047^{\mathrm{a}}$ \\
\hline $\mathrm{GFR}, \mathrm{mL} / \mathrm{min} / 1.73 \mathrm{~m}^{2}$ & $76.8 \pm 20.6$ & $73 \cdot 5 \pm 17.0$ & $81.2 \pm 22.5$ & 0.064 \\
\hline $\mathrm{LDH}, \mathrm{U} / \mathrm{L}$ & $562.6 \pm 324.8$ & $512.1 \pm 272.5$ & $604.4 \pm 360.6$ & 0.211 \\
\hline $\mathrm{CRP}, \mathrm{mg} / \mathrm{dL}$ & $3.1 \pm 3.9$ & $2.6 \pm 3.2$ & $3 \cdot 5 \pm 4 \cdot 5$ & 0.343 \\
\hline D-dimer, mg/L & $3.5 \pm 3.9$ & $2.9 \pm 4.1$ & $4.0 \pm 3.7$ & 0.241 \\
\hline
\end{tabular}

Values are presented as mean \pm SD or number (\%).

ECOG PS, Eastern Cooperative Oncology Group performance status; GFR, glomerular filtration rate; LDH, lactate dehydrogenase; CRP, C-reactive protein.

${ }^{a}$ Statistically significant $(p<0.05)$.

age and the K-GDS, and correlated positively with the EORTC-QLQ-C30 global health status and the physical and role functioning scales. Patients with a cognitive dysfunction identified by the K-MMSE had a lower educational level. K-GDS correlated negatively with the EORTC-QLQ-C30 scores for global health status, physical, role, emotional, and cognitive functioning scales. The scores for the physical functioning scales correlated positively with the level of albumin but negatively with the levels of LDH, CRP, and D-dimer (Supplementary Table 1).

\section{Factors associated with treatment interruption}

The results of the univariate and multivariate analyses to compare patients in the treatment interruption and continuation groups are listed in Table 3. The clinical and laboratory variables that were significantly associated with treatment interruption were $\geq 2$ sites of distant metastases and lower level of albumin. Patients with visceral organ metastases such as in the liver, lung, or peritoneum were more likely to be in the treatment interruption group compared with patients with non-visceral organ metastases. In the geriatric assessment, there were no differences in K-MBI, K-MMSE, and K-GDS between the continuation and interruption group. More patients in the treatment interruption group showed a depen- dent activity of K-IADL, but there was no statistically significant difference. The patients with treatment interruption showed the significantly lower scores for the EORTC-QLQ-C30 physical and role functioning scales and higher score for the EORTC-QLQ-C30 fatigue and appetite loss symptom scale compared to patients with treatment continuation.

In the multivariate analysis, the variable that was significantly associated with treatment interruption was a low score for the EORTC-QLQ-C30 physical functioning scale (odds ratio [OR], 1.020; 95\% confidence interval [CI], 1.002 to $1.039 ; p=0.030$ ), and $\geq 2$ sites of distant metastases (OR, 2.965; 95\% CI, 1.012 to 8.681; $p=0.047$ )

\section{Survival analyses}

Sixty-three of the 75 patients (84.0\%) died during the follow-up period, and the median OS of all patients was 7.6 months. Forty (97.6\%) and 23 patients (67.6\%) died in the treatment interruption and continuation groups, respectively. The median OS were 5.7 and 11.4 months in the treatment interruption and continuation groups, respectively. The significant factors related to OS identified in the univariate analysis are summarized in Table 4. In the multivariate analysis, patients with distant metastases in two or more organs, treatment interruption, lower scores for the EORTC-QLQ-C30 physical func- 
Table 2. Geriatric assessment of the patients

\begin{tabular}{|c|c|c|c|c|}
\hline Parameter & All patients $(\mathrm{n}=75)$ & Continuation $(n=34)$ & Interruption $(n=41)$ & $p$ value \\
\hline Activities of daily living (K-MBI, $\mathrm{n}=75)$ & & & & 0.720 \\
\hline Independent & $38(50.7)$ & $18(52.9)$ & $20(48.8)$ & \\
\hline Dependent & $37(49 \cdot 3)$ & $16(47 \cdot 1)$ & $21(51.2)$ & \\
\hline Instrumental activities of daily living (K-IADL, $\mathrm{n}=75)$ & & & & 0.064 \\
\hline Independent & $42(56.0)$ & $23(67.6)$ & $19(46.3)$ & \\
\hline Dependent & $33(44.0)$ & $11(32.4)$ & $22(53.7)$ & \\
\hline Cognitive impairment (K-MMSE, $\mathrm{n}=74$ ) & $22.0 \pm 5.0$ & $23.4 \pm 4.8$ & $21.2 \pm 5.0$ & 0.117 \\
\hline$>24 / 30$ & $29(39.2)$ & $16(47.0)$ & $13(32.5)$ & 0.201 \\
\hline$\leq 24 / 30$ & $45(60.8)$ & $18(53.0)$ & $27(67.5)$ & - \\
\hline Depressive disorder (K-GDS, $\mathrm{n}=74$ ) & $9.0 \pm 8.2$ & $8.8 \pm 8.6$ & $9.2 \pm 8.0$ & 0.837 \\
\hline$<16 / 30$ & $55(74 \cdot 3)$ & $25(73 \cdot 5)$ & $30(75 \cdot 0)$ & 0.885 \\
\hline$\geq 16 / 30$ & $19(25 \cdot 7)$ & $9(26.5)$ & $10(25.0)$ & - \\
\hline \multicolumn{5}{|l|}{ EORTC-QLQ-C3O (n = 75) } \\
\hline Global health status & $52.7 \pm 22.9$ & $56.6 \pm 21.4$ & $49.6 \pm 23.9$ & 0.186 \\
\hline \multicolumn{5}{|l|}{ Functioning scale } \\
\hline Physical & $62.9 \pm 28.8$ & $71.3 \pm 23.4$ & $55.8 \pm 31.2$ & $0.017^{\mathrm{a}}$ \\
\hline Role & $61.8 \pm 40.9$ & $72.0 \pm 34.7$ & $53.2 \pm 43 \cdot 9$ & $0.047^{\mathrm{a}}$ \\
\hline Emotional & $83.8 \pm 25.7$ & $88.0 \pm 21.3$ & $80.3 \pm 28.7$ & 0.187 \\
\hline Cognitive & $74.6 \pm 28.2$ & $73.9 \pm 26.5$ & $75.2 \pm 29.8$ & 0.853 \\
\hline Social & $83.0 \pm 28.8$ & $89.1 \pm 20.9$ & $78.0 \pm 33.4$ & 0.095 \\
\hline \multicolumn{5}{|l|}{ Symptom scale/item } \\
\hline Fatigue & $33.6 \pm 31.6$ & $25.1 \pm 26.3$ & $40.6 \pm 34.2$ & $0.034^{\mathrm{a}}$ \\
\hline Nausea and vomiting & $6.7 \pm 16.6$ & $6.8 \pm 19.6$ & $6.5 \pm 13.9$ & 0.936 \\
\hline Pain & $32.4 \pm 40.4$ & $24.0 \pm 38.5$ & $39.4 \pm 41.1$ & 0.098 \\
\hline Dyspnea & $15 \cdot 5 \pm 28.1$ & $10.7 \pm 19.5$ & $19 \cdot 5 \pm 33 \cdot 3$ & 0.181 \\
\hline Insomnia & $17.7 \pm 28.1$ & $21.5 \pm 30.5$ & $14.6 \pm 25.8$ & 0.300 \\
\hline Appetite loss & $41.7 \pm 44.5$ & $29.4 \pm 40.0$ & $52.0 \pm 45.9$ & $0.028^{a}$ \\
\hline Constipation & $32.8 \pm 38.9$ & $28.4 \pm 36.8$ & $36.5 \pm 40.7$ & 0.364 \\
\hline Diarrhea & $4.4 \pm 14.8$ & $3.9 \pm 10.8$ & $4.8 \pm 17.5$ & 0.773 \\
\hline Financial difficulties & $33.7 \pm 38.1$ & $31.3 \pm 36.6$ & $35.7 \pm 39.7$ & 0.624 \\
\hline
\end{tabular}

Values are presented as number (\%) or mean \pm SD.

K-MBI, Modified Bbarthel Index; K-IADL, Instrumental Activities of Daily Living; K-MMSE, Mini-Mental State Examination; K-GDS, Geriatric Depression Scale; EORTC-QLQ-C30, European Organisation for Research and Treatment of Cancer Qualityof-Life Core Questionnaire.

${ }^{\mathrm{a}}$ Statistically significant $(p<0.05)$.

tioning scales, and high level of D-dimer had a shorter OS (Table 5, Fig. 1).

\section{DISCUSSION}

When treating elderly patients with advanced cancer in oncology practice, the most commonly asked question is whether the patient can tolerate chemotherapy well and gain benefit from the chemotherapy. Some clinical trials have shown that the survival benefits of chemotherapy are similar between elderly and younger patients [10]. These benefits of chemotherapy are possible when the planned treatments are maintained. Several studies 
Table 3. Univariate and multivariate analyses comparing patients with treatment interruption versus treatment continuation

\begin{tabular}{|c|c|c|c|c|}
\hline \multirow{2}{*}{ Parameter } & \multicolumn{2}{|c|}{ Univariate } & \multicolumn{2}{|c|}{ Multivariate } \\
\hline & OR $(95 \% \mathrm{CI})$ & $p$ value & OR $(95 \% \mathrm{CI})$ & $p$ value \\
\hline$\geq 2$ Sites of distant metastases & $3.019(1.072-8.499)$ & 0.036 & $2.965(1.012-8.681)$ & 0.047 \\
\hline Albumin (below the median) & $2.288(0.975-5.369)$ & 0.057 & - & - \\
\hline Low score on physical function scale & $1.020(1.003-1.038)$ & 0.023 & $1.020(1.002-1.039)$ & 0.030 \\
\hline Low score on role function scale & $1.012(1.000-1.024)$ & 0.050 & - & - \\
\hline Low score on fatigue & $0.983(0.968-0.999)$ & 0.039 & - & - \\
\hline Low score on appetite loss symptom scale & $0.988(0.977-0.999)$ & 0.030 & - & - \\
\hline
\end{tabular}

OR, odds ratio; CI, confidence interval.

Table 4. Univariate survival analysis according to clinical, laboratory, and geriatric variables

\begin{tabular}{lcc}
\hline Parameter & OR $(95 \%$ CI $)$ & p value \\
\hline No. of distant metastases & $3.149(1.834-5.405)$ & 0.001 \\
Monochemotherapy & $2.662(1.523-4.655)$ & 0.001 \\
Interruption of treatment & $2.879(1.681-4.930)$ & 0.001 \\
Physical functioning score & $0.469(0.280-0.787)$ & 0.004 \\
Pain symptom score & $1.778(1.074-2.943)$ & 0.025 \\
Albumin & $0.577(0.349-0.955)$ & 0.032 \\
D-dimer & $2.808(1.645-4.796)$ & 0.001 \\
\hline
\end{tabular}

OR, odds ratio; CI, confidence interval.

Table 5. Multivariate survival analysis showing factors associated with overall survival

\begin{tabular}{|c|c|c|}
\hline Parameter & OR $(95 \% \mathrm{CI})$ & $p$ value \\
\hline $\begin{array}{l}\geq 2 \text { Sites of distant } \\
\text { metastases }\end{array}$ & $3.950(0.787-0.958)$ & 0.005 \\
\hline $\begin{array}{l}\text { Treatment, interruption vs. } \\
\text { continuation }\end{array}$ & $2.232(1.249-3.990)$ & 0.007 \\
\hline $\begin{array}{l}\text { Low physical functioning } \\
\text { score }\end{array}$ & $2.613(1.481-4.611)$ & 0.001 \\
\hline High level of D-dimer & $2.766(1.584-4.829)$ & 0.001 \\
\hline
\end{tabular}

OR, odds ratio; CI, confidence interval.

have been conducted to evaluate CGA as a predictor of chemotherapy toxicity. They showed that several elements of geriatric assessment predicted the occurrence of severe toxicity with chemotherapy. Previous studies have been performed in a disease-specific setting or heterogeneous group consisting of various treatment and stage. They also focused only on the role of CGA $[2,3,11]$. Our studies included only elderly patients with stage IV

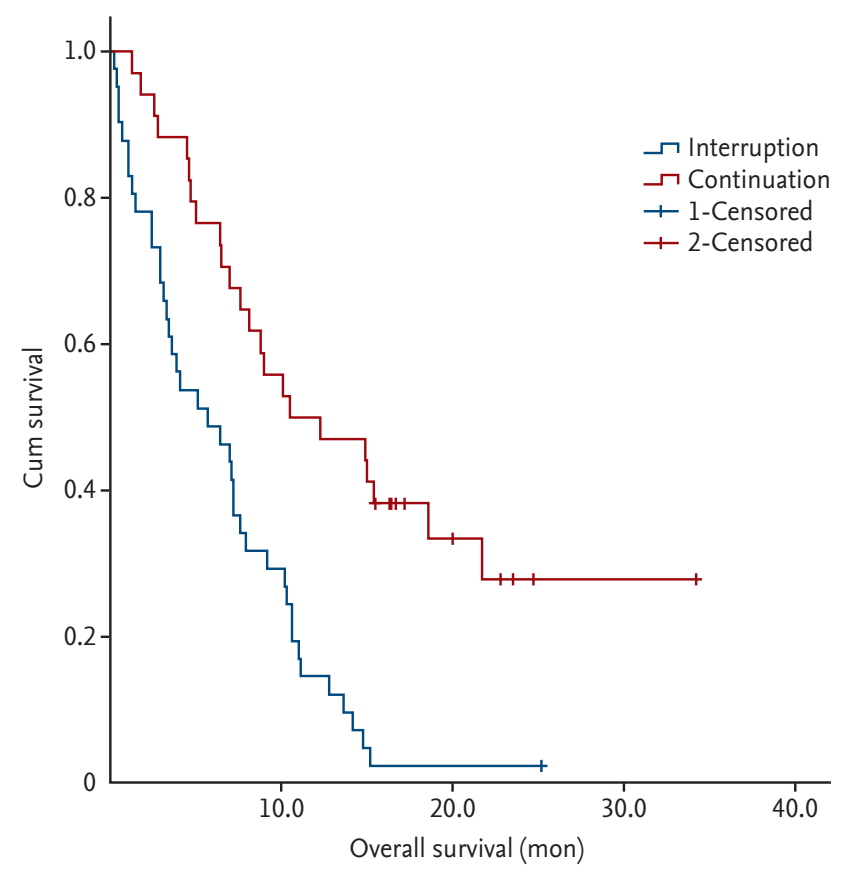

Figure 1. Kaplan-Meier survival curve according to treatment interruption. The patients with treatment interruption showed a significantly shorter overall survival compared with patients with treatment continuation.

cancer receiving chemotherapy and evaluated the quality of life and laboratory parameters at the time of treatment with CGA.

It is well documented that chronological age does not equate to physiological age, and an individual's functional age is far more important than chronological age [4]. The functional status is heterogeneous in elderly people. Thus, the selection of functionally fit elderly patients is important when deciding on treatment, and this process can be assisted with the inclusion of geriatric assessment $[4,12]$. Although the importance of geri- 
atric assessment is highlighted increasingly, geriatric assessment is not incorporated routinely into oncology practice because of the time and effort. The various components of geriatric assessment have been used in different studies $[3,4,11,12]$. The commonly adopted tools include assessment of: (1) function (activities of daily living, instrumental activities of daily living; (2) mobility (Timed Up-and-Go, falls in the past 6 months); (3) comorbidity; (4) nutrition; (5) social support; (6) cognition (MMSE); (7) psychological state (GDS); and (8) FRAIL scale (fatigue, resistance, ambulation, illnesses, and loss of weight) [13]. There are many limitations in conducing all of these CGA in real practice. To reduce the burden associated with additional assessments in practice, we used the demographic and clinical information available from the routine medical history and performed the relatively objective five simple questionnaires, which take about 25 minutes to complete.

In this study, 41 of 75 elderly patients did not receive the intended treatment consistently. Among the clinical variables, low body mass index and weight loss, which are known indicators of malnutrition, showed a trend of association with treatment interruption. Among the laboratory variables, low albumin level, which may indicate malnutrition, was also associated with treatment intolerance. These results are consistent with previous findings. Previous studies have shown that malnutrition has a negative impact on cancer patient compliance with oncology treatments as well as on survival and quality of life $[14,15]$.

The site and degree of metastases are prognostic factors in various cancers [16]. Visceral organ involvement and multiple distant metastases are associated with poor prognosis [16]. In our study, metastases to $\geq 2$ organs and visceral organ involvement such as in the liver, lung, and peritoneum were significantly associated with treatment interruption. This can be attributed to various symptoms and poor general conditions related to high metastatic tumor burden.

In the geriatric assessment, there were no significant associations between treatment interruption and activities of daily living (K-MBI), cognition (K-MMSE) or depression (K-GDS). The dependency of K-IADL showed a trend toward higher rate of treatment interruption in elderly patients with cancer.

The EORTC-QLQ-C30 physical, role functioning scores and fatigue, appetite loss symptom scores were significant predictors of treatment interruption. In the multivariate analyses, treatment interruption was independently associated with two factors: metastases to $\geq 2$ organs and physical functioning status assessed by the EORTC-QLQ-C3O. These results suggest that advanced disease status/high tumor burden and a poor physical functioning state may contribute to treatment intolerance in elderly patients with cancer.

An interesting point is the role of the EORTC-QLQ-C3O in assessing treatment tolerance of elderly patients. The EORTC-QLQ-C30 is often used to evaluate quality of life in patients with cancer, but is not commonly used for geriatric assessment. This scale comprises five simple questions and can be administered easily in clinical practice. Unexpectedly, we found that the physical functioning scale of the EORTC-QLQ-C30 was the predictor of treatment tolerance in elderly patients with cancer. However, this result should be interpreted cautiously because of small sample size and relatively small OR value. Further studies with more patients are necessary to confirm the usefulness of the EORTC-QLQ-C30 functioning scales in elderly patients with cancer.

In survival analyses, we confirmed that treatment interruption had a negative effect on overall survival. Therefore, improving treatment tolerance may lead to better patients' outcomes. We also found that patients with distant metastases to $\geq 2$ organs, low physical functioning score, and high level of D-dimer at diagnosis had a significantly shorter OS.

This study has some limitations. The sample size is small and the cancer types of the included patients were heterogeneous. Not all items that make up the CGA were evaluated, and the role of items such as mobility, functional muscle strength, and nutrition were not included in our analysis.

In conclusion, we identified two risk factors that were associated with vulnerability to first-line treatment on the basis of the assessment before starting treatment in elderly patients with cancer. These risk factors were metastases to $\geq 2$ organs and physical functional status assessed with the EORTC-QLQ-C30; these two variables indicate the metastatic tumor burden and the patient's functional status. Further research is required to evaluate the role of geriatric assessment including clinical characteristics and EORTC-QLQ-C30 in elderly patients with cancer. 


\section{KEY MESSAGE}

1. Some of elderly patients with cancer have shown intolerance during cancer treatment and they have not received the intended treatment.

2. Two or more sites of distant metastases among clinical variables and lower score for the European Organisation for Research and Treatment of Cancer Quality-of-Life Core Questionnaire (EORTC-QLQ-C30) physical functioning scale were significantly associated with treatment interruption.

3. Treatment interruption had a negative effect on overall survival in elderly patients with cancer (hazard ratio, 2.232; 95\% confidence interval, 1.249 to $3.990 ; p=0.007$ ).

\section{Conflict of interest}

No potential conflict of interest relevant to this article was reported.

\section{REFERENCES}

1. Kurita GP, Sjogren P, Ekholm O, et al. Prevalence and predictors of cognitive dysfunction in opioid-treated patients with cancer: a multinational study. J Clin Oncol 2011;29:1297-1303.

2. Extermann M, Boler I, Reich RR, et al. Predicting the risk of chemotherapy toxicity in older patients: the Chemotherapy Risk Assessment Scale for High-Age Patients (CRASH) score. Cancer 2012;118:3377-3386.

3. Hurria A, Togawa K, Mohile SG, et al. Predicting chemotherapy toxicity in older adults with cancer: a prospective multicenter study. J Clin Oncol 2011;29:3457-3465.

4. Wildiers H, Heeren P, Puts M, et al. International Society of Geriatric Oncology consensus on geriatric assessment in older patients with cancer. J Clin Oncol 2014;32:25952603.

5. Jung HY, Park BK, Shin HS, et al. Development of the Korean version of Modified Barthel Index (K-MBI): multi-center study for subjects with stroke. J Korean Acad Rehabil Med 2007;31:283-297.
6. Han C, Jo SA, Jo I, Kim E, Park MH, Kang Y. An adaptation of the Korean Mini-Mental State Examination (K-MMSE) in elderly Koreans: demographic influence and population-based norms (the AGE study). Arch Gerontol Geriatr 2008;47:302-310.

7. Bae JN, Cho MJ. Development of the Korean version of the Geriatric Depression Scale and its short form among elderly psychiatric patients. J Psychosom Res 2004;57:297305.

8. Yun YH, Park YS, Lee ES, et al. Validation of the Korean version of the EORTC QLQ-C30. Qual Life Res 2004;13:863-868.

9. Folstein MF, Folstein SE, McHugh PR. "Mini-mental state": a practical method for grading the cognitive state of patients for the clinician. J Psychiatr Res 1975;12:189198.

10. Gridelli C, Balducci L, Ciardiello F, et al. Treatment of elderly patients with non-small-cell lung cancer: results of an international expert panel meeting of the Italian Association of Thoracic Oncology. Clin Lung Cancer 2015;16:325-333.

11. Pal SK, Katheria V, Hurria A. Evaluating the older patient with cancer: understanding frailty and the geriatric assessment. CA Cancer J Clin 2010;60:120-132.

12. Caillet P, Canoui-Poitrine F, Vouriot J, et al. Comprehensive geriatric assessment in the decision-making process in elderly patients with cancer: ELCAPA study. J Clin Oncol 2011;29:3636-3642.

13. Jung HW, Yoo HJ, Park SY, et al. The Korean version of the FRAIL scale: clinical feasibility and validity of assessing the frailty status of Korean elderly. Korean J Intern Med 2016;31:594-600.

14. Bozzetti F. Why the oncologist should consider the nutritional status of the elderly cancer patient. Nutrition 2015;31:590-593.

15. Jung YH, Woo IS, Han CW. Clinical characteristics and outcomes in diffuse large B cell lymphoma patients aged 70 years and older: a single-center experience with a literature review. Korean J Intern Med 2015;30:684-693.

16. Yardley DA. Visceral disease in patients with metastatic breast cancer: efficacy and safety of treatment with ixabepilone and other chemotherapeutic agents. Clin Breast Cancer 2010;10:64-73. 


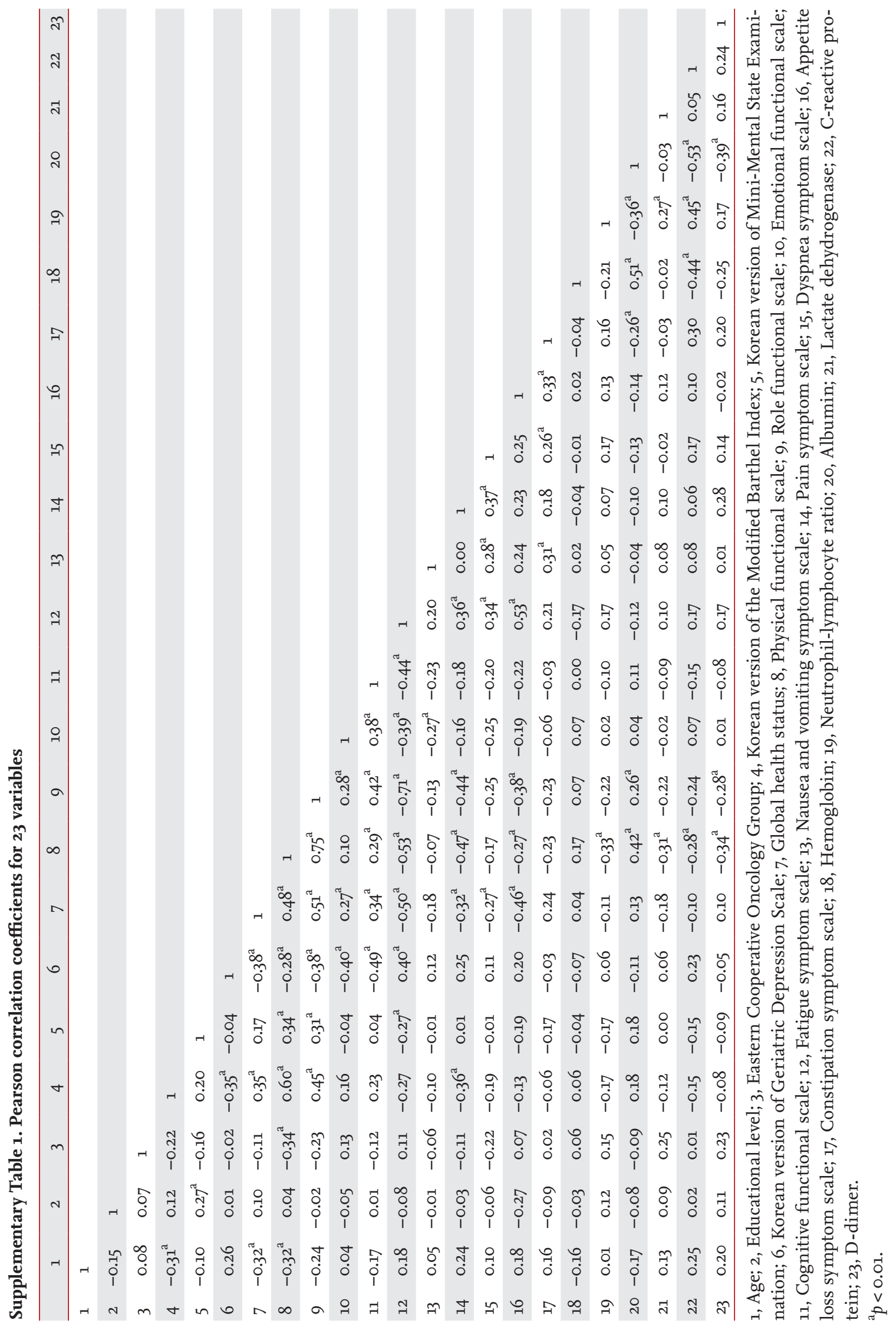

